\title{
Fixed point results of weakly contraction mappings in partially ordered $b$-metric spaces
}

\author{
K. Kalyani ${ }^{1}$ and N. Seshagiri Rao ${ }^{2^{*}}$ (])
}

\begin{abstract}
Objectives: We explored the results of fixed point, coincidence point and coupled coincidence point for the mappings in an ordered metric spaces. Our results generalized and extended the well-known results in the literature. Some numerical examples are provided for justifying the results obtained.

Result: Some fixed point results are found for a self mapping in a partially ordered $b$-metric space which satisfies a generalized week contraction condition. Furthermore, these results are extended for two self mappings for obtaining coincidence point, coupled coincidence point and coupled common fixed point in the same context. A few examples are presented to support the findings.
\end{abstract}

Keywords: $(\hat{\phi}, \hat{\psi})$-Weak contractions, Fixed point, Coincidence point, Compatible mappings, Coupled coincidence point, Coupled common fixed points

Mathematics Subject Classification: Primary: 54H25, Secondary: $47 \mathrm{H} 10$

\section{Introduction}

A $b$-metric space also referred to as a metric type space by some researchers is one of the most influential generalizations of ordinary metric space. It has a wide range of uses in mathematical research and scientific applications. It was first established by Bakhtin in [7] and eventually expanded upon by Czerwik in [10]. Later, Aghajani et al. [1], Allahyari et al. [5] investigated some fixed point results of generalized contractive mappings in partially ordered $b$-metric space and then applied their results to quadratic integral equations. Common fixed point results for generalized weak contractions in the same context was studied by Aghajani et al. [2]. Also, the results on common fixed point for two self mappings under an implicit relation was explored by Akkouchi [3]. Some remarks on fixed point results in $b$-metric space were discussed by Aleksić et al. [4]. Common fixed point for weak $\varphi$-contractions on $b$-metric spaces was examined by Aydi et al. [6]. Recently, some results on fixed point, coincidence point, coupled coincidence point for the self mappings satisfying generalized weak contractions have been discussed by Belay et al. [8], Seshagiri Rao et al. [20, $24,25]$ in partially ordered $b$-metric space with necessary topological properties.

In this paper, we introduced the following generalized weak contraction condition which involve the altering distance functions $\hat{\phi} \in \hat{\Phi}, \hat{\psi} \in \hat{\Psi}$ defined below to acquire a fixed point of a mapping $\mathscr{L}: \mathfrak{P} \rightarrow \mathfrak{P}$ in a partially ordered $b$-metric space

$$
\hat{\phi}(\Im \partial(\mathscr{L} \zeta, \mathscr{L} \varpi)) \leq \hat{\phi}(\mathcal{C}(\zeta, \varpi))-\hat{\psi}(\mathcal{D}(\zeta, \varpi)),
$$

for any $\zeta, \varpi \in \mathfrak{P}$ with $\zeta \preceq \varpi$, $>1$ and, where

\footnotetext{
*Correspondence: seshu.namana@gmail.com

2 Department of Applied Mathematics, School of Applied Natural

Sciences, Adama Science and Technology University, Post Box No.1888

Adama, Ethiopia

Full list of author information is available at the end of the article
}

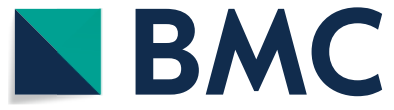

(c) The Author(s) 2022. Open Access This article is licensed under a Creative Commons Attribution 4.0 International License, which permits use, sharing, adaptation, distribution and reproduction in any medium or format, as long as you give appropriate credit to the original author(s) and the source, provide a link to the Creative Commons licence, and indicate if changes were made. The images or other third party material in this article are included in the article's Creative Commons licence, unless indicated otherwise in a credit line to the material. If material is not included in the article's Creative Commons licence and your intended use is not permitted by statutory regulation or exceeds the permitted use, you will need to obtain permission directly from the copyright holder. To view a copy of this licence, visit http://creativecommons.org/licenses/by/4.0/. The Creative Commons Public Domain Dedication waiver (http://creativeco mmons.org/publicdomain/zero/1.0/) applies to the data made available in this article, unless otherwise stated in a credit line to the data. 


$$
\mathcal{C}(\zeta, \varpi)=\max \left\{\frac{\partial(\varpi, \mathscr{L} \varpi)[1+\partial(\zeta, \mathscr{L} \zeta)]}{1+\partial(\zeta, \varpi)}, \frac{\partial(\zeta, \mathscr{L} \zeta) \partial(\varpi, \mathscr{L} \varpi)}{1+\partial(\zeta, \varpi)}, \partial(\zeta, \mathscr{L} \zeta), \partial(\varpi, \mathscr{L} \varpi), \partial(\zeta, \varpi)\right\}
$$

and

$$
\mathcal{D}(\zeta, \varpi)=\max \left\{\frac{\partial(\varpi, \mathscr{L} \varpi)[1+\partial(\zeta, \mathscr{L} \zeta)]}{1+\partial(\zeta, \varpi)}, \partial(\zeta, \varpi)\right\} .
$$

Also generalized this result by involving two self mappings in the above generalized contraction condition to obtain a coincidence point, coupled coincidence point and common fixed point for the mappings in the same context. Our results are generalized and extended the results of Belay et al. [8], Bhaskar et al. [9], Harjani et al. [12] and Jachymski [14] and Seshagiri Rao et al. [20, 24, 25]. The authors may refer the papers of Aghajani et al. [1], Dorić et al. [11], Huaping Huang et al. [13], Roshan et al. $[18,19]$ and Seshagiri Rao et al. [21-23] for the basic definitions and necessary results which we used in the present study.

In the whole paper, we use the following nations for the altering distance functions: $\hat{\Phi}=\{\hat{\phi} / \hat{\phi}$ is continuous, non-decrasing self mapping on $[0,+\infty)$ with $\hat{\phi}(\varepsilon)=0$ iff $\varepsilon=0$, for $\varepsilon \in[0,+\infty)\}$ and $\hat{\Psi}=\{\hat{\psi} / \hat{\psi}$ is lower semi-continuous self mapping on $[0,+\infty)$ such that $\hat{\psi}(\varepsilon)=0$ if and only if $\varepsilon=0$, where $\varepsilon \in[0,+\infty)\}$.

\section{Main results}

We start this section with the following fixed point result in a complete partially ordered $b$-metric space.

Theorem 2.1 Suppose $(\mathfrak{P}, \check{\partial}, \preceq)$ be a complete partially ordered b-metric space with $s>1$. Assume that a continuous self mapping $\mathscr{L}$ on $\mathfrak{P}$ is non-decreasing with respect to $\preceq$ and satisfies the condition (1). If for some $\zeta_{0} \in \mathfrak{P}$ such that $\zeta_{0} \preceq \mathscr{L} \zeta_{0}$, then $\mathscr{L}$ has a fixed point in $\mathfrak{P}$.

Proof The proof is trivial for $\mathscr{L} \zeta_{0}=\zeta_{0}$, for some $\zeta_{0} \in \mathfrak{P}$. Suppose not then $\zeta_{0} \prec \mathscr{L} \zeta_{0}$. Now define a sequence $\left\{\zeta_{n}\right\} \subset \mathfrak{P}$ by $\zeta_{n+1}=\mathscr{L} \zeta_{n}$, for $n \geq 0$. Since $\mathscr{L}$ is non-decreasing then

$$
\zeta_{0} \prec \mathscr{L} \zeta_{0}=\zeta_{1} \preceq \cdots \preceq \zeta_{n} \preceq \mathscr{L} \zeta_{n}=\zeta_{n+1} \preceq \cdots .
$$

If for some $n_{0} \in \mathbb{N}, \zeta_{n_{0}}=\zeta_{n_{0}+1}$, then from (2), $\mathscr{L}$ has a fixed point $\zeta_{n_{0}}$. Assume that $\zeta_{n} \neq \zeta_{n+1}$ for all $n \geq 1$. Since $\zeta_{n}>\zeta_{n-1}$ for all $n \geq 1$, then from (1), we have

$$
\begin{aligned}
\hat{\phi}\left(ð\left(\zeta_{n}, \zeta_{n+1}\right)\right)= & \hat{\phi}\left(\partial\left(\mathscr{L} \zeta_{n-1}, \mathscr{L} \zeta_{n}\right)\right) \\
& \leq \hat{\phi}\left(\circlearrowleft \partial\left(\mathscr{L} \zeta_{n-1}, \mathscr{L} \zeta_{n}\right)\right) \\
& \leq \hat{\phi}\left(\mathcal{C}\left(\zeta_{n-1}, \zeta_{n}\right)\right) \\
& -\hat{\psi}\left(\mathcal{D}\left(\zeta_{n-1}, \zeta_{n}\right)\right) .
\end{aligned}
$$

Thus from (3), we have

$$
\partial\left(\zeta_{n}, \zeta_{n+1}\right)=\partial\left(\mathscr{L} \zeta_{n-1}, \mathscr{L} \zeta_{n}\right) \leq \frac{1}{\varsigma} \mathcal{C}\left(\zeta_{n-1}, \zeta_{n}\right),
$$

where

$$
\begin{aligned}
\mathcal{C}\left(\zeta_{n-1}, \zeta_{n}\right)= & \max \left\{\frac{\partial\left(\zeta_{n}, \mathscr{L} \zeta_{n}\right)\left[1+\partial\left(\zeta_{n-1}, \mathscr{L} \zeta_{n-1}\right)\right]}{1+\partial\left(\zeta_{n-1}, \zeta_{n}\right)},\right. \\
& \frac{\partial\left(\zeta_{n-1}, \mathscr{L} \zeta_{n-1}\right) \partial\left(\zeta_{n}, \mathscr{L} \zeta_{n}\right)}{1+\partial\left(\zeta_{n-1}, \zeta_{n}\right)}, \\
& \partial\left(\zeta_{n-1}, \mathscr{L} \zeta_{n-1}\right), \\
= & \max \left\{\partial\left(\zeta_{n}, \zeta_{n+1}\right),\right. \\
& \left.\frac{\partial\left(\zeta_{n-1}, \zeta_{n}\right) \partial\left(\zeta_{n}, \zeta_{n+1}\right)}{1+\partial\left(\zeta_{n-1}, \zeta_{n}\right)}, \partial\left(\zeta_{n-1}, \zeta_{n}\right)\right\} \\
\leq & \max \left\{\partial\left(\zeta_{n}, \zeta_{n+1}\right), \partial\left(\zeta_{n-1}, \zeta_{n}\right)\right\} .
\end{aligned}
$$

If $\max \left\{\partial\left(\zeta_{n}, \zeta_{n+1}\right), \partial\left(\zeta_{n-1}, \zeta_{n}\right)\right\}=\partial\left(\zeta_{n}, \zeta_{n+1}\right)$ for some $n \geq 1$, then from (4), we have

$$
\partial\left(\zeta_{n}, \zeta_{n+1}\right) \leq \frac{1}{\jmath} \partial\left(\zeta_{n}, \zeta_{n+1}\right),
$$

this is a contradiction. Hence, $\max \left\{\partial\left(\zeta_{n}, \zeta_{n+1}\right), \partial\left(\zeta_{n-1}, \zeta_{n}\right)\right\}$ $=\partial\left(\zeta_{n-1}, \zeta_{n}\right)$ for all $n \geq 1$. Thus from (4) we have

$$
\partial\left(\zeta_{n}, \zeta_{n+1}\right) \leq \frac{1}{\sigma} \partial\left(\zeta_{n-1}, \zeta_{n}\right) .
$$

Since $\frac{1}{s} \in(0,1)$ then $\left\{\zeta_{n}\right\}$ is a Cauchy sequence from $[4$, 6]. Also, the completeness of $\mathfrak{P}$ implies that $\zeta_{n} \rightarrow \varepsilon$ for some $\varepsilon \in \mathfrak{P}$.

Furthermore the continuity of $\mathscr{L}$ implies that,

$$
\mathscr{L} \varepsilon=\mathscr{L}\left(\lim _{n \rightarrow+\infty} \zeta_{n}\right)=\lim _{n \rightarrow+\infty} \mathscr{L} \zeta_{n}=\lim _{n \rightarrow+\infty} \zeta_{n+1}=\varepsilon,
$$


which shows that $\mathscr{L}$ has a fixed point $\varepsilon \in \mathfrak{P}$.

We have the following result in which the mapping $\mathscr{L}$ is not continuous, still is valid to have a fixed point.

Theorem 2.2 According to Theorem 2.1, a non-continuous self mapping $\mathscr{L}$ has a fixed point if $\mathfrak{P}$ meets the condition (6):

a non-decreasing sequence $\left\{\zeta_{n}\right\} \subseteq \mathfrak{P}$ such that

$\zeta_{n} \rightarrow \varepsilon \in \mathfrak{P}$ then $\zeta_{n} \preceq \varepsilon$ for $n \in \mathbb{N}$,

that is, $\varepsilon=\sup \zeta_{n}$.

Proof As from Theorem 2.1, a non-decreasing Cauchy sequence $\left\{\zeta_{n}\right\} \subseteq \mathfrak{P}$ exists such that $\zeta_{n} \rightarrow \varepsilon \in \mathfrak{P}$. Hence from condition (6), $\zeta_{n} \preceq \varepsilon$ for all $n$, i.e., $\varepsilon=\sup \zeta_{n}$.

Next to show that $\varepsilon$ is a fixed point of $\mathscr{L}$ in $\mathfrak{P}$. Suppose that $\mathscr{L} \varepsilon \neq \varepsilon$. Let

$$
\begin{aligned}
\mathcal{C}\left(\zeta_{n}, \varepsilon\right)= & \max \left\{\frac{\partial(\varepsilon, \mathscr{L} \varepsilon)\left[1+\partial\left(\zeta_{n}, \mathscr{L} \zeta_{n}\right)\right]}{1+\partial\left(\zeta_{n}, \varepsilon\right)}\right. \\
& , \frac{\partial\left(\zeta_{n}, \mathscr{L} \zeta_{n}\right) \partial(\varepsilon, \mathscr{L} \varepsilon)}{1+\partial\left(\zeta_{n}, \varepsilon\right)}, \\
& \left.\partial\left(\zeta_{n}, \mathscr{L} \zeta_{n}\right), \partial(\varepsilon, \mathscr{L} \varepsilon), \partial\left(\zeta_{n}, \varepsilon\right)\right\}
\end{aligned}
$$

and

$$
\mathcal{D}\left(\zeta_{n}, \varepsilon\right)=\max \left\{\frac{\partial(\varepsilon, \mathscr{L} \varepsilon)\left[1+\partial\left(\zeta_{n}, \mathscr{L} \zeta_{n}\right)\right]}{1+\partial\left(\zeta_{n}, \varepsilon\right)}, \partial\left(\zeta_{n}, \varepsilon\right)\right\} .
$$

As $n \rightarrow+\infty$ and the fact that $\lim _{n \rightarrow+\infty} \zeta_{n}=\varepsilon$, we obtain that

$$
\lim _{n \rightarrow+\infty} \mathcal{C}\left(\zeta_{n}, \varepsilon\right)=\max \{\partial(\varepsilon, \mathscr{L} \varepsilon), 0\}=\partial(\varepsilon, \mathscr{L} \varepsilon),
$$

and

$$
\lim _{n \rightarrow+\infty} \mathcal{D}\left(\zeta_{n}, \varepsilon\right)=\max \{\partial(\varepsilon, \mathscr{L} \varepsilon), 0\}=\partial(\varepsilon, \mathscr{L} \varepsilon) .
$$

Since $\zeta_{n} \preceq \varepsilon$ for any $n$, then (1) becomes

$$
\begin{aligned}
\hat{\phi}\left(\partial\left(\zeta_{n+1}, \mathscr{L} \varepsilon\right)\right)= & \hat{\phi}\left(\partial\left(\mathscr{L} \zeta_{n}, \mathscr{L} \varepsilon\right)\right) \\
& \leq \hat{\phi}\left(\sigma \partial\left(\mathscr{L} \zeta_{n}, \mathscr{L} \varepsilon\right)\right. \\
& \leq \hat{\phi}\left(\mathcal{C}\left(\zeta_{n}, \varepsilon\right)\right) \\
& -\hat{\psi}\left(\mathcal{D}\left(\zeta_{n}, \varepsilon\right)\right) .
\end{aligned}
$$

Taking $n \rightarrow+\infty$ in (9) and from Eqs. (7) and (8), we get

$$
\hat{\phi}(ð(\varepsilon, \mathscr{L} \varepsilon)) \leq \hat{\phi}(ð(\varepsilon, \mathscr{L} \varepsilon))-\hat{\psi}(ð(\varepsilon, \mathscr{L} \varepsilon))<\hat{\phi}(ð(\varepsilon, \mathscr{L} \varepsilon)),
$$

which is a contradiction. Hence, $\mathscr{L} \varepsilon=\varepsilon$, i.e., $\mathscr{L}$ has a fixed point $\varepsilon$ in $\mathfrak{P}$.
Theorem 2.3 If every two elements of $\mathfrak{P}$ are comparable then $\mathscr{L}$ has a unique fixed point in Theorems 2.1 and 2.2.

Proof Let $\zeta^{*} \neq \varpi^{*}$ be two fixed points of $\mathscr{L}$ in $\mathfrak{P}$, then from (1), we have

$$
\begin{aligned}
& \hat{\phi}\left(\partial\left(\mathscr{L} \zeta^{*}, \mathscr{L} \varpi^{*}\right)\right) \leq \hat{\phi}\left(\circlearrowleft \partial\left(\mathscr{L} \zeta^{*}, \mathscr{L} \varpi^{*}\right)\right) \\
& \quad \leq \hat{\phi}\left(\mathcal{C}\left(\zeta^{*}, \varpi^{*}\right)\right)-\hat{\psi}\left(\mathcal{D}\left(\zeta^{*}, \varpi^{*}\right)\right) .
\end{aligned}
$$

As a result, we get

$$
ð\left(\zeta^{*}, \varpi^{*}\right)=ð\left(\mathscr{L} \zeta^{*}, \mathscr{L} \varpi^{*}\right) \leq \frac{1}{\jmath} \mathcal{C}\left(\zeta^{*}, \varpi^{*}\right),
$$

where

$$
\begin{aligned}
\mathcal{C}\left(\zeta^{*}, \varpi^{*}\right)= & \max \left\{\frac{\partial\left(\varpi^{*}, \mathscr{L} \varpi^{*}\right)\left[1+\partial\left(\zeta^{*}, \mathscr{L} \zeta^{*}\right)\right]}{1+\partial\left(\zeta^{*}, \varpi^{*}\right)},\right. \\
& \frac{\partial\left(\zeta^{*}, \mathscr{L} \zeta^{*}\right) \partial\left(\varpi^{*}, \mathscr{L} \varpi^{*}\right)}{1+\partial\left(\zeta^{*}, \varpi^{*}\right)}, \partial\left(\zeta^{*}, \mathscr{L} \zeta^{*}\right), \\
& \left.\partial\left(\varpi^{*}, \mathscr{L} \varpi^{*}\right), \partial\left(\zeta^{*}, \varpi^{*}\right)\right\} \\
= & \max \left\{\frac{\partial\left(\varpi^{*}, \varpi^{*}\right)\left[1+\partial\left(\zeta^{*}, \zeta^{*}\right)\right]}{1+\partial\left(\zeta^{*}, \varpi^{*}\right)},\right. \\
& \frac{\partial\left(\zeta^{*}, \zeta^{*}\right) \partial\left(\varpi^{*}, \varpi^{*}\right)}{1+\partial\left(\zeta^{*}, \varpi^{*}\right)}, \partial\left(\zeta^{*}, \zeta^{*}\right) \\
& \left., \partial\left(\varpi^{*}, \varpi^{*}\right), \partial\left(\zeta^{*}, \varpi^{*}\right)\right\} \\
= & \max \left\{0, \partial\left(\zeta^{*}, \varpi^{*}\right)\right\} \\
= & \partial\left(\zeta^{*}, \varpi^{*}\right) .
\end{aligned}
$$

Therefore from (10), we have

$$
\partial\left(\zeta^{*}, \varpi^{*}\right) \leq \frac{1}{\sigma} \partial\left(\zeta^{*}, \varpi^{*}\right)<\partial\left(\zeta^{*}, \varpi^{*}\right),
$$

which leads contradiction to $\zeta^{*} \neq \varpi^{*}$. Thus, $\zeta^{*}=\varpi^{*}$.

We have the following consequences from Theorems 2.1, 2.2 and 2.3.

Corollary 2.4 Instead $\mathcal{D}(\zeta, \varpi)$ by $\mathcal{C}(\zeta, \varpi)$ in condition (1), we have the same conclusions as from Theorems 2.1, 2.2 and 2.3 .

Corollary 2.5 Taking $\hat{\phi}(m)=m$ and $\hat{\psi}(m)=(1-k) m$ in Corollary 2.4, then the contraction condition becomes

$$
\begin{gathered}
\partial(\mathscr{L} \zeta, \mathscr{L} \varpi) \leq \frac{k}{\sigma} \max \left\{\frac{\partial(\varpi, \mathscr{L} \varpi)[1+\partial(\zeta, \mathscr{L} \zeta)]}{1+\partial(\zeta, \varpi)},\right. \\
\left.\frac{\partial(\zeta, \mathscr{L} \zeta) \partial(\varpi, \mathscr{L} \varpi)}{1+\partial(\zeta, \varpi)}, \partial(\zeta, \mathscr{L} \zeta), \partial(\varpi, \mathscr{L} \varpi), \partial(\zeta, \varpi)\right\} .
\end{gathered}
$$

Then one can arrive at the same conclusions as in Theorems 2.1, 2.2 and 2.3 . 
A self mapping $\mathscr{L}$ on $\mathfrak{P}$ with respect to $f: \mathfrak{P} \rightarrow \mathfrak{P}$ is a generalized contraction mapping, if it satisfies the following condition for all $\zeta, \varpi \in \mathfrak{P}$ with $f \zeta \preceq f \varpi$, $\hat{\phi} \in \hat{\Phi}$ and $\hat{\psi} \in \hat{\Psi}$ :

$$
\hat{\phi}(๘ \partial(\mathscr{L} \zeta, \mathscr{L} \varpi)) \leq \hat{\phi}\left(\mathcal{C}_{f}(\zeta, \varpi)\right)-\hat{\psi}\left(\mathcal{D}_{f}(\zeta, \varpi)\right),
$$

where

$$
\begin{aligned}
\mathcal{C}_{f}(\zeta, \varpi)= & \max \left\{\frac{\partial(f \varpi, \mathscr{L} \varpi)[1+\partial(f \zeta, \mathscr{L} \zeta)]}{1+\partial(f \zeta, f \varpi)},\right. \\
& \frac{\partial(f \zeta, \mathscr{L} \zeta) \partial(f \varpi, \mathscr{L} \varpi)}{1+\partial(f \zeta, f \varpi)}, \partial(f \zeta, \mathscr{L} \zeta), \\
& \partial(f \varpi, \mathscr{L} \varpi), \partial(f \zeta, f \varpi)\},
\end{aligned}
$$

and

$$
\begin{aligned}
& \mathcal{D}_{f}(\zeta, \varpi) \\
& =\max \left\{\frac{\partial(f \omega, \mathscr{L} \varpi)[1+\partial(f \zeta, \mathscr{L} \zeta)]}{1+\partial(f \zeta, f \varpi)}, \partial(f \zeta, f \varpi)\right\} .
\end{aligned}
$$

Now, we have the following result.

Theorem 2.6 The two continuous self-mappings $\mathscr{L}$, $f$ on $\mathfrak{P}$ have a coincidence point, if they satisfy the following conditions:

a. $\mathscr{L}$ is a monotone f-non-decreasing,

b. $\mathscr{L} \mathfrak{P} \subseteq \mathscr{f} \mathfrak{P}$ and a pair ( $\mathscr{L}, f$ ) are compatible,

c. $f \zeta_{0} \leq \mathscr{L} \zeta_{0}$ for some $\zeta_{0} \in \mathfrak{P}$ and

d. satisfies the condition (11) in a complete partially ordered b-metric space ( $\mathfrak{P}, ð, \preceq)$.

Proof From Theorem 2.2 of [5], we have the sequences $\left\{\zeta_{n}\right\},\left\{\varpi_{n}\right\} \subseteq \mathfrak{P}$ with

$$
\varpi_{n}=\mathscr{L} \zeta_{n}=f \quad \zeta_{n+1} \text { for all } n \geq 0,
$$

for which

$$
f \quad \zeta_{0} \preceq f \quad \zeta_{1} \preceq \cdots \preceq f \quad \zeta_{n} \preceq f \quad \zeta_{n+1} \preceq \cdots .
$$

Now from [5], we have to show that

$$
\partial\left(\varpi_{n}, \varpi_{n+1}\right) \leq \lambda \partial\left(\varpi_{n-1}, \varpi_{n}\right),
$$

for all $n \geq 1$ and where $\lambda \in\left[0, \frac{1}{\sigma}\right)$.

From Eqs. (11), (14) and (15), we have

$$
\begin{aligned}
\hat{\phi}\left(\varsigma \partial\left(\varpi_{n}, \varpi_{n+1}\right)\right) & =\hat{\phi}\left(\varsigma \partial\left(\mathscr{L} \zeta_{n}, \mathscr{L} \zeta_{n+1}\right)\right) \\
& \leq \hat{\phi}\left(\mathcal{C}_{f}\left(\zeta_{n}, \zeta_{n+1}\right)\right) \\
& -\hat{\psi}\left(\mathcal{D}_{f}\left(\zeta_{n}, \zeta_{n+1}\right)\right)
\end{aligned}
$$

where

$$
\begin{aligned}
\mathcal{C}_{f}\left(\zeta_{n}, \zeta_{n+1}\right)= & \max \left\{\frac{\partial\left(f \zeta_{n+1}, \mathscr{L} \zeta_{n+1}\right)\left[1+\partial\left(f \zeta_{n}, \mathscr{L} \zeta_{n}\right)\right]}{1+\partial\left(f \zeta_{n}, f \zeta_{n+1}\right)},\right. \\
& \frac{\partial\left(f \zeta_{n}, \mathscr{L} \zeta_{n}\right) \partial\left(f \zeta_{n+1}, \mathscr{L} \zeta_{n+1}\right)}{1+\partial\left(f \zeta_{n}, f \zeta_{n+1}\right)}, \\
& \left.\partial\left(f \zeta_{n}, \mathscr{L} \zeta_{n}\right), \partial\left(f \zeta_{n+1}, \mathscr{L} \zeta_{n+1}\right), \partial\left(f \zeta_{n}, f \zeta_{n+1}\right)\right\} \\
= & \max \left\{\frac{\partial\left(\varpi_{n}, \varpi_{n+1}\right)\left[1+\partial\left(\varpi_{n-1}, \varpi_{n}\right)\right]}{1+\partial\left(\varpi_{n-1}, \varpi_{n}\right)},\right. \\
& \frac{\partial\left(\varpi_{n-1}, \varpi_{n}\right) \partial\left(\varpi_{n}, \varpi_{n+1}\right)}{1+\partial\left(\varpi_{n-1}, \varpi_{n}\right)}, \\
& \left.\partial\left(\varpi_{n-1}, \varpi_{n}\right), \partial\left(\varpi_{n}, \varpi_{n+1}\right), \partial\left(\varpi_{n-1}, \varpi_{n}\right)\right\} \\
& \leq \max \left\{\partial\left(\varpi_{n-1}, \varpi_{n}\right), \partial\left(\varpi_{n}, \varpi_{n+1}\right)\right\}
\end{aligned}
$$

and

$$
\begin{aligned}
\mathcal{D}_{f}\left(\zeta_{n}, \zeta_{n+1}\right)= & \max \left\{\frac{\partial\left(f \zeta_{n+1}, \mathscr{L} \zeta_{n+1}\right)\left[1+\partial\left(f \zeta_{n}, \mathscr{L} \zeta_{n}\right)\right]}{1+\partial\left(f \zeta_{n}, f \zeta_{n+1}\right)},\right. \\
& \left.\partial\left(f \zeta_{n}, \mathcal{f} \zeta_{n+1}\right)\right\} \\
= & \max \left\{\frac{\partial\left(\varpi_{n}, \varpi_{n+1}\right)\left[1+\partial\left(\varpi_{n-1}, \varpi_{n}\right)\right]}{1+\partial\left(\varpi_{n-1}, \varpi_{n}\right)},\right. \\
& \left.\partial\left(\varpi_{n-1}, \varpi_{n}\right)\right\} \\
= & \max \left\{\partial\left(\varpi_{n-1}, \varpi_{n}\right), \partial\left(\varpi_{n}, \varpi_{n+1}\right)\right\} .
\end{aligned}
$$

From Eq. (17), we have

$$
\begin{aligned}
& \hat{\phi}\left(\sigma \quad \partial\left(\varpi_{n}, \varpi_{n+1}\right)\right) \leq \hat{\phi}\left(\max \left\{\partial\left(\varpi_{n-1}, \varpi_{n}\right), \partial\left(\varpi_{n}, \varpi_{n+1}\right)\right\}\right) \\
& \quad-\hat{\psi}\left(\max \left\{\partial\left(\varpi_{n-1}, \varpi_{n}\right), \partial\left(\varpi_{n}, \varpi_{n+1}\right)\right\}\right) .
\end{aligned}
$$

If $0<\partial\left(\varpi_{n-1}, \varpi_{n}\right) \leq ð\left(\varpi_{n}, \varpi_{n+1}\right)$ for some $n$, then Eq. (18) follows that

$$
\begin{aligned}
& \hat{\phi}\left(\circlearrowleft \partial\left(\varpi_{n}, \varpi_{n+1}\right)\right) \leq \hat{\phi}\left(\partial\left(\varpi_{n}, \varpi_{n+1}\right)\right) \\
& -\hat{\psi}\left(\partial\left(\varpi_{n}, \varpi_{n+1}\right)\right)<\hat{\phi}\left(\partial\left(\varpi_{n}, \varpi_{n+1}\right)\right),
\end{aligned}
$$

or equivalently

$$
\text { ऽ } \partial\left(\varpi_{n}, \varpi_{n+1}\right) \leq \partial\left(\varpi_{n}, \varpi_{n+1}\right),
$$

a contradiction. Therefore, from Eq. (18) we have

$$
\text { ऽ } \partial\left(\varpi_{n}, \varpi_{n+1}\right) \leq \partial\left(\varpi_{n-1}, \varpi_{n}\right) .
$$

Hence, $\lambda \in\left[0, \frac{1}{\sigma}\right.$ ) from (16). According to Lemma 3.1 of [15] and from Eq. (16), we have 


$$
\lim _{n \rightarrow+\infty} \mathscr{L} \zeta_{n}=\lim _{n \rightarrow+\infty} \mathscr{f} \zeta_{n+1}=\mu, \text { for } \mu \in \mathfrak{P} .
$$

From condition (b), we have

$$
\lim _{n \rightarrow+\infty} \partial\left(f\left(\mathscr{L} \zeta_{n}\right), \mathscr{L}\left(f \zeta_{n}\right)\right)=0,
$$

and the continuity of $\mathscr{L}$ and $f$ we have,

$$
\lim _{n \rightarrow+\infty} f\left(\mathscr{L} \zeta_{n}\right)=f \mu, \quad \lim _{n \rightarrow+\infty} \mathscr{L}\left(f \zeta_{n}\right)=\mathscr{L} \mu .
$$

Furthermore,

$$
\begin{aligned}
& \frac{1}{\sigma} \partial(\mathscr{L} \mu, f \mu) \leq \partial\left(\mathscr{L} \mu, \mathscr{L}\left(f \zeta_{n}\right)\right)+\sigma \partial\left(\mathscr{L}\left(f \zeta_{n}\right),\right. \\
& \left.f\left(\mathscr{L} \zeta_{n}\right)\right)+\sigma \partial\left(f\left(\mathscr{L} \zeta_{n}\right), f \mu\right) .
\end{aligned}
$$

Thus, $\partial(\mathscr{L} v, f v)=0$ as $n \rightarrow+\infty$ in (22) and hence the result.

We have the following result without the continuity property of $f$ and $\mathscr{L}$ in Theorem 2.6.

Theorem 2.7 If $\mathfrak{P}$ has the property in Theorem 2.6 that a sequence $\left\{f \zeta_{n}\right\} \subset \mathfrak{P}$ is a non-decreasing such that $\lim _{n \rightarrow+\infty} f \zeta_{n}=f \quad \zeta \in f \mathfrak{P}$, and

$f \mathfrak{P} \subseteq \mathfrak{P}$ is closed and $f \zeta_{n} \preceq f \zeta$, $f \zeta \preceq$ of $\zeta$ ) for $n$ and $\mathscr{f} \zeta_{0} \preceq \mathscr{L} \zeta_{0}$ for some $\zeta_{0} \in \mathfrak{P}$,

then the weakly compatible mappings $\mathscr{L}$, f have a coincidence point. Besides that, when $\mathscr{L}$ and $f$ commute at their coincidence points, then $\mathscr{L}$, f have a common fixed point in $\mathfrak{P}$.

Proof As from Theorem 2.6, $\left\{\varpi_{n}\right\}=\left\{\mathscr{L} \zeta_{n}\right\}=\left\{f \zeta_{n+1}\right\}$ is a Cauchy sequence. Since of $\mathfrak{P}$ is closed then

$$
\lim _{n \rightarrow+\infty} \mathscr{L} \zeta_{n}=\lim _{n \rightarrow+\infty} f \zeta_{n+1}=f \quad \mu \text { for } \mu \in \mathfrak{P} .
$$

Thus, $f \quad \zeta_{n} \preceq f \mu$ for all $n$. Next to show that $\mathscr{L}$, $f$ have a coincidence point $\mu$. From (11), we have

$$
\hat{\phi}\left(\Im \partial\left(\mathscr{L} \zeta_{n}, \mathscr{L} \zeta\right)\right) \leq \hat{\phi}\left(\mathcal{C}_{f}\left(\zeta_{n}, \zeta\right)\right)-\hat{\psi}\left(\mathcal{D}_{f}\left(\zeta_{n}, \zeta\right)\right),
$$

where

$$
\begin{aligned}
\mathcal{C}_{f}\left(\zeta_{n}, \mu\right)= & \max \left\{\frac{\partial(f \mu, \mathscr{L} \mu)\left[1+\partial\left(f \zeta_{n}, \mathscr{L} \zeta_{n}\right)\right]}{1+\partial\left(f \zeta_{n}, f \mu\right)},\right. \\
& \frac{\partial\left(f \zeta_{n}, \mathscr{L} \zeta_{n}\right) \partial(f \mu, \mathscr{L} \mu)}{1+\partial\left(f \zeta_{n}, f \mu\right)}, \partial\left(f \zeta_{n}, \mathscr{L} \zeta_{n}\right), \\
& \left.\partial(f \mu, \mathscr{L} \mu), \partial\left(f \zeta_{n}, f \mu\right)\right\} \\
\rightarrow & \max \{\partial(f \mu, \mathscr{L} \mu), 0,0, \partial(f \mu, \mathscr{L} \mu), 0\} \\
= & \partial(f \mu, \mathscr{L} \mu) \text { as } n \rightarrow+\infty,
\end{aligned}
$$

and

$$
\begin{aligned}
\mathcal{D}_{f}\left(\zeta_{n}, \mu\right)= & \max \left\{\frac{\partial(f \mu, \mathscr{L} \mu)\left[1+\partial\left(f \zeta_{n}, \mathscr{L} \zeta_{n}\right)\right]}{1+\partial\left(f \zeta_{n}, f \mu\right)},\right. \\
& \left.\partial\left(f \zeta_{n}, f \mu\right)\right\} \\
& \rightarrow \max \{\partial(f \mu, \mathscr{L} \mu), 0\} \\
& =\partial(f \mu, \mathscr{L} \mu) \text { as } n \rightarrow+\infty .
\end{aligned}
$$

Thus Eq. (23) becomes

$$
\begin{aligned}
& \hat{\phi}\left(s \lim _{n \rightarrow+\infty} \partial\left(\mathscr{L} \zeta_{n}, \mathscr{L} \zeta\right)\right) \leq \hat{\phi}(\partial(f \mu, \mathscr{L} \mu)) \\
& -\hat{\psi}(\delta(f \mu, \mathscr{L} \mu))<\hat{\phi}(\delta(f \mu, \mathscr{L} \mu)) .
\end{aligned}
$$

As a result, we have

$$
\lim _{n \rightarrow+\infty} \partial\left(\mathscr{L} \zeta_{n}, \mathscr{L} \zeta\right)<\frac{1}{\sigma} \partial(f \mu, \mathscr{L} \mu) .
$$

Furthermore, the triangular inequality of $ð$, we have

$$
\frac{1}{\sigma} \partial(f \mu, \mathscr{L} \mu) \leq \partial\left(f \mu, \mathscr{L} \zeta_{n}\right)+\partial\left(\mathscr{L} \zeta_{n}, \mathscr{L} \mu\right),
$$

thus Eqs. (25) and (26) lead to contradiction, if of $\mu \neq \mathscr{L} \mu$. Hence, of $\mu=\mathscr{L} \mu$. Let $f \mu=\mathscr{L} \mu=\rho$, then $\quad \mathscr{L} \rho=\mathscr{L}(f \mu)=f(\mathscr{L} \mu)=$ of $\rho$. Since of $\mu=f(f \mu)=f \rho$, then by Eq. (23) with ff $\mu=\mathscr{L} \mu$ and $f \quad \rho=\mathscr{L} \rho$, we get

$$
\begin{aligned}
& \hat{\phi}(\varsigma \quad \partial(\mathscr{L} \mu, \mathscr{L} \rho)) \leq \hat{\phi}\left(\mathcal{C}_{f}(\mu, \rho)\right) \\
& -\hat{\psi}\left(\mathcal{D}_{f}(\mu, \rho)\right)<\hat{\phi}(\partial(\mathscr{L} \mu, \mathscr{L} \rho)),
\end{aligned}
$$

or equivalently,

$$
\text { ऽ } \partial(\mathscr{L} \mu, \mathscr{L} \rho) \leq ð(\mathscr{L} \mu, \mathscr{L} \rho),
$$

which is a contradiction, if $\mathscr{L} \mu \neq \mathscr{L} \rho$. Thus, $\mathscr{L} \mu=\mathscr{L} \rho=\rho$ and implies that $\mathscr{L} \mu=f \quad \rho=\rho$. Hence the result. 
Definition 2.8 Consider the partially ordered $b$-metric space $(\mathfrak{P}, ð, \preceq)$. A mapping $\mathscr{L}: \mathfrak{P} \times \mathfrak{P} \rightarrow \mathfrak{P}$ is a generalized $(\hat{\phi}, \hat{\psi})$-contractive mapping with respect to a self mapping $f$ on $\mathfrak{P}$, if

$$
\begin{aligned}
& \phi\left({ }^{k}{ } \partial(\mathscr{L}(\zeta, \varpi), \mathscr{L}(\varrho, \sigma))\right) \\
& \leq \hat{\phi}\left(\mathcal{C}_{f}(\zeta, \varpi, \varrho, \sigma)\right) \\
& -\hat{\psi}\left(\mathcal{D}_{f}(\zeta, \varpi, \varrho, \sigma)\right),
\end{aligned}
$$

for all $\zeta, \varpi, \varrho, \sigma \in \mathfrak{P}$ with $f \zeta \preceq f \quad \varrho$ and $f \varpi \succeq f \sigma$, $k>2$, $s>1, \hat{\phi} \in \hat{\Phi}, \hat{\psi} \in \hat{\Psi}$ and where

$$
\begin{aligned}
\mathcal{C}_{f}(\zeta, \varpi, \varrho, \sigma)= & \max \left\{\frac{\partial(f \varrho, \mathscr{L}(\varrho, \sigma))[1+\partial(f \zeta, \mathscr{L}(\zeta, \varpi))]}{1+\partial(f \zeta, f \varrho)},\right. \\
& \frac{\partial(f \zeta \mathscr{L}(\zeta, \varpi)) \partial(f \varrho, \mathscr{L}(\varrho, \sigma))}{1+\partial(f \zeta, f \varrho)}, \\
& \partial(f \zeta, \mathscr{L}(\zeta, \varpi)), \partial(f \varrho, \mathscr{L}(\varrho, \sigma)), \\
& \partial(f \zeta, f \varrho)\},
\end{aligned}
$$

and

$$
\begin{aligned}
\mathcal{D}_{f}(\zeta, \varpi, \varrho, \sigma)= & \max \\
& \left\{\frac{\partial(f \varrho, \mathscr{L}(\varrho, \sigma))[1+\partial(f \quad \zeta, \mathscr{L}(\zeta, \varpi))]}{1+\partial(f \zeta, f \varrho)},\right. \\
& \partial(f \zeta, f \varrho)\} .
\end{aligned}
$$

Theorem 2.9 Let $(\mathfrak{P}, ð, \preceq)$ be a complete partially ordered b-metric space. Assume that a mapping $\mathscr{L}: \mathfrak{P} \times \mathfrak{P} \rightarrow \mathfrak{P}$ satisfies the condition (28) and, $\mathscr{L}, \mathscr{f}$ are continuous, $\mathscr{L}$ has mixed f-monotone property and commutes with of . Suppose, if for some $\left(\zeta_{0}, \varpi_{0}\right) \in \mathfrak{P} \times \mathfrak{P}$ such that of $\zeta_{0} \preceq \mathscr{L}\left(\zeta_{0}, \varpi_{0}\right)$, ff $\varpi_{0} \succeq \mathscr{L}\left(\varpi_{0}, \zeta_{0}\right)$ and $\mathscr{L}(\mathfrak{P} \times \mathfrak{P}) \subseteq f(\mathfrak{P})$, then $\mathscr{L}$ and $f$ have a coupled coincidence point in $\mathfrak{P}$.

Proof From Theorem 2.2 of [5], there will be two sequences $\left\{\zeta_{n}\right\},\left\{\varpi_{n}\right\} \subset \mathfrak{P}$ such that

$$
f \zeta_{n+1}=\mathscr{L}\left(\zeta_{n}, \varpi_{n}\right), \quad f \varpi_{n+1}=\mathscr{L}\left(\varpi_{n}, \zeta_{n}\right) \text {, for all } n \geq 0 \text {. }
$$

In particular, the sequences $\left\{f \zeta_{n}\right\}$ and $\left\{f \varpi_{n}\right\}$ are non-decreasing and non-increasing in $\mathfrak{P}$. Put $\zeta=\zeta_{n}, \varpi=\varpi_{n}, \varrho=\zeta_{n+1}, \sigma=\varpi_{n+1}$ in (28), we get

$$
\begin{aligned}
& \hat{\phi}\left(\sigma{ }^{k} \partial\left(f \zeta_{n+1}, f \zeta_{n+2}\right)\right) \\
& =\hat{\phi}\left(\sigma k \partial\left(\mathscr{L}\left(\zeta_{n}, \varpi_{n}\right), \mathscr{L}\left(\zeta_{n+1}, \varpi_{n+1}\right)\right)\right) \\
& \quad \leq \hat{\phi}\left(\mathcal{C}_{f}\left(\zeta_{n}, \varpi_{n}, \zeta_{n+1}, \varpi_{n+1}\right)\right) \\
& \quad-\hat{\psi}\left(\mathcal{D}_{f}\left(\zeta_{n}, \varpi_{n}, \zeta_{n+1}, \varpi_{n+1}\right)\right),
\end{aligned}
$$

where

$$
\begin{aligned}
& \mathcal{C}_{f}\left(\zeta_{n}, \varpi_{n}, \zeta_{n+1}, \varpi_{n+1}\right) \\
& \leq \max \left\{\partial\left(f \quad \zeta_{n}, f \quad \zeta_{n+1}\right),\right. \\
& \left.\partial\left(f \quad \zeta_{n+1}, f \quad \zeta_{n+2}\right)\right\}
\end{aligned}
$$

and

$$
\begin{aligned}
& \mathcal{D}_{f}\left(\zeta_{n}, \varpi_{n}, \zeta_{n+1}, \varpi_{n+1}\right) \\
& =\max \left\{\partial\left(f \quad \zeta_{n}, f \quad \zeta_{n+1}\right), \partial\left(f \quad \zeta_{n+1}, f \quad \zeta_{n+2}\right)\right\} .
\end{aligned}
$$

Therefore from (29), we have

$$
\begin{aligned}
\hat{\phi}\left(\sigma{ }^{k} \partial\left(f \zeta_{n+1}, f \zeta_{n+2}\right)\right) \leq & \hat{\phi}\left(\operatorname { m a x } \left\{\partial\left(f \zeta_{n}, f \zeta_{n+1}\right),\right.\right. \\
& \left.\left.\partial\left(f \quad \zeta_{n+1}, f \quad \zeta_{n+2}\right)\right\}\right) \\
& -\hat{\psi}\left(\operatorname { m a x } \left\{\partial\left(f \quad \zeta_{n}, f \quad \zeta_{n+1}\right),\right.\right. \\
& \left.\left.\partial\left(f \quad \zeta_{n+1}, f \zeta_{n+2}\right)\right\}\right) .
\end{aligned}
$$

Similarly by taking $\zeta=\varpi_{n+1}, \varpi=\zeta_{n+1}, \varrho=\zeta_{n}, \sigma=\zeta_{n}$ in (28), we get

$$
\begin{aligned}
& \hat{\phi}\left(\sigma \quad k \partial\left(f \varpi_{n+1}, f \varpi_{n+2}\right)\right) \\
& \leq \hat{\phi}\left(\operatorname { m a x } \left\{\partial\left(f \varpi_{n}, f \varpi_{n+1}\right),\right.\right. \\
& \left.\left.\partial\left(f \varpi_{n+1}, f \varpi_{n+2}\right)\right\}\right) \\
& -\hat{\psi}\left(\operatorname { m a x } \left\{\partial\left(f \varpi_{n}, f \varpi_{n+1}\right),\right.\right. \\
& \left.\left.\partial\left(f \varpi_{n+1}, f \varpi_{n+2}\right)\right\}\right) .
\end{aligned}
$$

We know that $\max \left\{\hat{\phi}\left(\varepsilon_{1}\right), \hat{\phi}\left(\varepsilon_{2}\right)\right\}=\hat{\phi}\left\{\max \left\{\varepsilon_{1}, \varepsilon_{2}\right\}\right\}$ for $\varepsilon_{1}, \varepsilon_{2} \in[0,+\infty)$. Then by adding Eqs. (32) and (33) together to get,

$$
\begin{aligned}
\hat{\phi}\left(\sigma{ }^{k} \delta_{n}\right) \leq & \phi\left(\operatorname { m a x } \left\{\partial\left(f \quad \zeta_{n}, f \quad \zeta_{n+1}\right),\right.\right. \\
& \partial\left(f \quad \zeta_{n+1}, f \quad \zeta_{n+2}\right), \partial\left(f \quad \varpi_{n}, f \varpi_{n+1}\right), \\
& \left.\left.\partial\left(f \varpi_{n+1}, f \varpi_{n+2}\right)\right\}\right) \\
& -\hat{\psi}\left(\operatorname { m a x } \left\{\partial\left(f \quad \zeta_{n}, f \quad \zeta_{n+1}\right),\right.\right. \\
& \partial\left(f \quad \zeta_{n+1}, f \quad \zeta_{n+2}\right), \partial\left(f \quad \varpi_{n}, f \varpi_{n+1}\right), \\
& \left.\left.\partial\left(f \varpi_{n+1}, f \varpi_{n+2}\right)\right\}\right)
\end{aligned}
$$

where

$$
\delta_{n}=\max \left\{\partial\left(f \quad \zeta_{n+1}, f \quad \zeta_{n+2}\right), \partial\left(f \varpi_{n+1}, f \varpi_{n+2}\right)\right\} .
$$

Let us denote,

$$
\begin{aligned}
\nabla_{n}= & \max \left\{\partial\left(f \quad \zeta_{n}, f \quad \zeta_{n+1}\right), \partial\left(f \quad \zeta_{n+1}, f \zeta_{n+2}\right),\right. \\
& \left.\partial\left(f \quad \varpi_{n}, f \quad \varpi_{n+1}\right), \partial\left(f \quad \varpi_{n+1}, f \quad \varpi_{n+2}\right)\right\} .
\end{aligned}
$$

Hence from Eqs. (32)-(35), we obtain that 


$$
{ }^{k} \delta_{n} \leq \nabla_{n}
$$

Now to claim that

$$
\delta_{n} \leq \lambda \delta_{n-1},
$$

for $n \geq 1$ and $\lambda=\frac{1}{\sigma k} \in[0,1)$.

Suppose that if $\nabla_{n}=\delta_{n}$ then from (37), we get ${ }^{k} \delta_{n} \leq \delta_{n}$ this leads to $\delta_{n}=0$ since $s>1$ and thus (38) holds. Suppose $\nabla_{n}=\max \left\{\partial\left(f \zeta_{n}, f \quad \zeta_{n+1}\right)\right.$, ठ(f $\left.\left.\varpi_{n}, f \varpi_{n+1}\right)\right\}$, that is, $\nabla_{n}=\delta_{n-1}$ thence (37) follows (38).

Now, we can deduce from (37) that $\delta_{n} \leq \lambda^{n} \delta_{0}$ and therefore,

$$
\partial\left(f \zeta_{n+1}, f \zeta_{n+2}\right) \leq \lambda^{n} \delta_{0} \text { and } \partial\left(f \varpi_{n+1} \text {, f } \varpi_{n+2}\right) \leq \lambda^{n} \delta_{0} \text {, }
$$

which shows that $\left\{f \zeta_{n}\right\}$ and $\left\{f \varpi_{n}\right\}$ in $\mathfrak{P}$ are Cauchy sequences from Lemma 3.1 of [15]. Therefore, we can conclude from [3] of Theorem 2.2 that $\mathscr{L}$ and $f$ in $\mathfrak{P}$ have a coincidence point.

Corollary 2.10 Suppose ( $\mathfrak{P}, ð, \preceq)$ be a complete partially ordered b-metric space. Let a continuous mapping $\mathscr{L}: \mathfrak{P} \times \mathfrak{P} \rightarrow \mathfrak{P}$ has a mixed monotone property and satisfies the contraction conditions below for any $\zeta, \varpi, \varrho, \sigma \in \mathfrak{P}$ such that $\zeta \preceq \varrho$ and $\varpi \succeq \sigma, k>2$, 。 $>1$, $\hat{\phi} \in \hat{\Phi}$ and $\hat{\psi} \in \hat{\Psi}$ :

i.

$$
\begin{aligned}
& \hat{\phi}\left(\sigma{ }^{k} \partial(\mathscr{L}(\zeta, \varpi), \mathscr{L}(\varrho, \sigma))\right) \\
& \leq \hat{\phi}\left(\mathcal{C}_{f}(\zeta, \varpi, \varrho, \sigma)\right)-\hat{\psi}\left(\mathcal{D}_{f}(\zeta, \varpi, \varrho, \sigma)\right),
\end{aligned}
$$

ii.

$$
\begin{aligned}
& \partial(\mathscr{L}(\zeta, \varpi), \mathscr{L}(\varrho, \sigma)) \leq \frac{1}{\sigma k} \mathcal{C}_{f}(\zeta, \varpi, \varrho, \sigma) \\
& -\frac{1}{\sigma k} \hat{\psi}\left(\mathcal{D}_{f}(\zeta, \varpi, \varrho, \sigma)\right) .
\end{aligned}
$$

where

$$
\begin{aligned}
\mathcal{C}_{f}(\zeta, \varpi, \varrho, \sigma)= & \max \\
& \left\{\frac{\partial(\varrho, \mathscr{L}(\varrho, \sigma))[1+\partial(\zeta, \mathscr{L}(\zeta, \varpi))]}{1+\partial(\zeta, \varrho)},\right. \\
& \frac{\partial(\zeta, \mathscr{L}(\zeta, \varpi)) \partial(\varrho, \mathscr{L}(\varrho, \sigma))}{1+\partial(\zeta, \varrho)} \\
& \partial(\zeta, \mathscr{L}(\zeta, \varpi)), \partial(\varrho, \mathscr{L}(\varrho, \sigma)), \partial(\zeta, \varrho)\},
\end{aligned}
$$

and

$$
\begin{aligned}
\mathcal{D}_{f}(\zeta, \varpi, \varrho, \sigma)= & \max \\
& \left\{\frac{\partial(\varrho, \mathscr{L}(\varrho, \sigma))[1+\partial(\zeta, \mathscr{L}(\zeta, \varpi))]}{1+\partial(\zeta, \varrho)},\right. \\
& \partial(\zeta, \varrho)\} .
\end{aligned}
$$

If there exists $\left(\zeta_{0}, \varpi_{0}\right) \in \mathfrak{P} \times \mathfrak{P}$ such that $\zeta_{0} \preceq \mathscr{L}\left(\zeta_{0}, \varpi_{0}\right)$ and $\varpi_{0} \succeq \mathscr{L}\left(\varpi_{0}, \zeta_{0}\right)$, then $\mathscr{L}$ has a coupled fixed point in $\mathfrak{P}$.

Theorem 2.11 A unique coupled common fixed point for $\mathscr{L}$ and $f$ exists in Theorem 2.9, if for every $(\zeta, \varpi),(k, \ell) \in \mathfrak{P} \times \mathfrak{P}$ there is some $\left(\alpha^{*}, \beta^{*}\right) \in \mathfrak{P} \times \mathfrak{P}$ such that $\left(\mathscr{L}\left(\alpha^{*}, \beta^{*}\right), \mathscr{L}\left(\beta^{*}, \alpha^{*}\right)\right)$ is comparable to $(\mathscr{L}(\zeta, \varpi), \mathscr{L}(\varpi, \zeta))$ and to $(\mathscr{L}(k, \ell), \mathscr{L}(\ell, k))$.

Proof From Theorem 2.9, the mappings $\mathscr{L}$ and $f$ have a coupled coincidence point in $\mathfrak{P}$. Let $(\zeta, \varpi),(k, \ell) \in \mathfrak{P} \times \mathfrak{P}$ are two coupled coincidence points of $\mathscr{L}$ and $f$. Now to claim that $f \zeta=f k$ and ff $\varpi=f \ell$. By hypotheses $\left(\mathscr{L}\left(\alpha^{*}, \beta^{*}\right), \mathscr{L}\left(\beta^{*}, \alpha^{*}\right)\right)$ is comparable to $(\mathscr{L}(\zeta, \varpi), \mathscr{L}(\varpi, \zeta))$ for some $\left(\alpha^{*}, \beta^{*}\right) \in \mathfrak{P} \times \mathfrak{P}$.

Now, assume the following

$$
\begin{aligned}
& (\mathscr{L}(\zeta, \varpi), \mathscr{L}(\varpi, \zeta)) \leq\left(\mathscr{L}\left(\alpha^{*}, \beta^{*}\right), \mathscr{L}\left(\beta^{*}, \alpha^{*}\right)\right) \text { and } \\
& (\mathscr{L}(k, \ell), \mathscr{L}(\ell, k)) \leq\left(\mathscr{L}\left(\alpha^{*}, \beta^{*}\right), \mathscr{L}\left(\beta^{*}, \alpha^{*}\right)\right) .
\end{aligned}
$$

Suppose $\alpha^{*}{ }_{0}=\alpha^{*}$ and $\beta^{*}{ }_{0}=\beta^{*}$ then there is a point $\left(\alpha^{*}{ }_{1}, \beta^{*}{ }_{1}\right) \in \mathfrak{P} \times \mathfrak{P}$ such that

$$
\text { f } \alpha^{*}{ }_{1}=\mathscr{L}\left(\alpha^{*}{ }_{0}, \beta^{*}{ }_{0}\right) \text {, f } \beta^{*}{ }_{1}=\mathscr{L}\left(\beta^{*}{ }_{0}, \alpha^{*}{ }_{0}\right) \quad(n \geq 1) \text {. }
$$

We have the sequences $\left\{f \alpha^{*}{ }_{n}\right\}$ and $\left\{f \beta^{*}{ }_{n}\right\}$ in $\mathfrak{P}$ as by the repeated application of the above argument with

$$
\text { f } \alpha^{*}{ }_{n+1}=\mathscr{L}\left(\alpha^{*}{ }_{n}, \beta^{*}{ }_{n}\right) \text {, ff } \beta^{*}{ }_{n+1}=\mathscr{L}\left(\beta^{*}{ }_{n}, \alpha^{*}{ }_{n}\right), n \geq 0 \text {. }
$$

Similarly, define the sequences $\left\{f \zeta_{n}\right\},\left\{f \varpi_{n}\right\}$ and $\left\{f k_{n}\right\}$, $\left\{f \ell_{n}\right\}$ in $\mathfrak{P}$ by setting $\zeta_{0}=\zeta, \varpi_{0}=\varpi$ and $k_{0}=k, \ell_{0}=\ell$. Furthermore, we have

$$
\begin{aligned}
f \quad \zeta_{n} & \rightarrow \mathscr{L}(\zeta, \varpi), \text { ff } \varpi_{n} \rightarrow \mathscr{L}(\varpi, \zeta) \text {, f } k_{n} \\
& \rightarrow \mathscr{L}(k, \ell), \text { f } \ell_{n} \rightarrow \mathscr{L}(\ell, k)(n \geq 1) .
\end{aligned}
$$

Therefore by induction, we have

$$
\left(f \quad \zeta_{n}, f \varpi_{n}\right) \leq\left(f \alpha^{*}{ }_{n} \text {,f } \beta^{*}{ }_{n}\right), n \geq 0 \text {. }
$$

Now from Eq. (28), we get 


$$
\begin{aligned}
& \hat{\phi}\left(\partial\left(f \quad \zeta, f \alpha^{*}{ }_{n+1}\right)\right) \leq \hat{\phi}\left(\sigma{ }^{k} \partial\left(f \quad \zeta, f \alpha^{*}{ }_{n+1}\right)\right) \\
& =\hat{\phi}\left(\sigma{ }^{k} \partial\left(\mathscr{L}(\zeta, \varpi), \mathscr{L}\left(\alpha^{*}{ }_{n}, \beta^{*}{ }_{n}\right)\right)\right) \\
& \quad \leq \hat{\phi}\left(\mathcal{C}_{f}\left(\zeta, \varpi, \alpha^{*}{ }_{n}, \beta^{*}{ }_{n}\right)\right) \\
& -\hat{\psi}\left(\mathcal{D}_{f}\left(\zeta, \varpi, \alpha^{*}{ }_{n}, \beta^{*}{ }_{n}\right)\right),
\end{aligned}
$$

where

$$
\begin{aligned}
\mathcal{C} f & \left(\zeta, \varpi, \alpha^{*}{ }_{n}, \beta^{*}{ }_{n}\right) \\
= & \max \left\{\frac{\partial\left(f \alpha^{*}{ }_{n}, \mathscr{L}\left(\alpha^{*}{ }_{n}, \beta^{*}{ }_{n}\right)\right)[1+\partial(f \zeta, \mathscr{L}(\zeta, \varpi))]}{1+\partial\left(f \zeta, f \alpha^{*}{ }_{n}\right)},\right. \\
& \frac{\partial(f \zeta, \mathscr{L}(\zeta, \varpi)) \partial\left(f \alpha^{*}{ }_{n}, \mathscr{L}\left(\alpha^{*}{ }_{n}, \beta^{*}{ }_{n}\right)\right)}{1+\partial\left(f \zeta, f \alpha^{*}{ }_{n}\right)}, \\
& \partial(f \zeta, \mathscr{L}(\zeta, \varpi)), \partial\left(f \alpha^{*}{ }_{n}, \mathscr{L}\left(\alpha^{*}{ }_{n}, \beta^{*}{ }_{n}\right)\right), \\
& \left.\partial\left(f \zeta, f \alpha^{*}{ }_{n}\right)\right\} \\
= & \max \left\{0, \partial\left(f \zeta, f \alpha^{*}{ }_{n}\right)\right\} \\
= & \partial\left(f \zeta, f \alpha^{*}{ }_{n}\right)
\end{aligned}
$$

and

$$
\begin{aligned}
\mathcal{D}_{f}\left(\zeta, \varpi, \alpha^{*}{ }_{n}, \beta^{*}{ }_{n}\right) & =\max \left\{\frac{\partial\left(f \alpha^{*}{ }_{n}, \mathscr{L}\left(\alpha^{*}{ }_{n}, \beta^{*}{ }_{n}\right)\right)[1+\partial(f \zeta, \mathscr{L}(\zeta, \varpi))]}{1+\partial\left(f \zeta, f \alpha^{*}{ }_{n}\right)}, \partial\left(f \zeta, f \alpha^{*}{ }_{n}\right)\right\} \\
& =\partial\left(f \zeta, f \alpha^{*}{ }_{n}\right) .
\end{aligned}
$$

As a result of Eq. (42), we now have

$$
\begin{aligned}
& \hat{\phi}\left(\partial\left(f \quad \zeta, f \quad \alpha^{*}{ }_{n+1}\right)\right) \leq \hat{\phi}\left(\partial\left(f \quad \zeta \text {, } f \quad \alpha^{*}{ }_{n}\right)\right) \\
& -\hat{\psi}\left(\partial\left(f \quad \zeta, f \quad \alpha^{*}{ }_{n}\right)\right) .
\end{aligned}
$$

As a consequence of a similar argument, we deduce that

$$
\begin{aligned}
& \hat{\phi}\left(\partial\left(f \quad \varpi, f \beta^{*}{ }_{n+1}\right)\right) \leq \hat{\phi}\left(\partial\left(f \quad \varpi, f \beta^{*}{ }_{n}\right)\right) \\
& -\hat{\psi}\left(\partial\left(f \varpi, f \beta^{*}{ }_{n}\right)\right) .
\end{aligned}
$$

Therefore from (43) and (44), we have

$$
\begin{aligned}
& \hat{\phi}\left(\max \left\{\partial\left(f \quad \zeta, f \quad \alpha^{*}{ }_{n+1}\right), \partial\left(f \quad \varpi, f \quad \beta^{*}{ }_{n+1}\right)\right\}\right) \\
& \leq \hat{\phi}\left(\max \left\{\partial\left(f \quad \zeta \text {,f } \quad \alpha^{*}{ }_{n}\right), \partial\left(f \quad \varpi, f \quad \beta^{*}{ }_{n}\right)\right\}\right) \\
& -\hat{\psi}\left(\max \left\{\partial\left(f \quad \zeta, f \quad \alpha^{*}{ }_{n}\right), \partial\left(f \quad \varpi, f \quad \beta^{*}{ }_{n}\right)\right\}\right) \\
& <\hat{\phi}\left(\max \left\{\partial\left(f \quad \zeta \text {,f } \quad \alpha^{*}{ }_{n}\right), \partial\left(f \quad \varpi, f \quad \beta^{*}{ }_{n}\right)\right\}\right) .
\end{aligned}
$$

The property of $\hat{\phi}$ implies that,

$$
\begin{aligned}
& \max \left\{\partial\left(f \quad \zeta, f \quad \alpha^{*}{ }_{n+1}\right), \partial\left(f \quad \varpi, f \quad \beta^{*}{ }_{n+1}\right)\right\} \\
& \quad<\max \left\{\partial\left(f \quad \zeta, f \quad \alpha^{*}{ }_{n}\right), \partial\left(f \quad \varpi, f \quad \beta^{*}{ }_{n}\right)\right\} .
\end{aligned}
$$

Hence, $\max \left\{\partial\left(f \zeta\right.\right.$, f $\left.\left.\alpha^{*}{ }_{n}\right), \partial\left(f \quad \varpi, f \beta^{*}{ }_{n}\right)\right\}$ is bounded below decreasing sequence of positive reals and by a result, we get $\lim _{n \rightarrow+\infty} \max \left\{\partial\left(f \quad \zeta, f \quad \alpha^{*}{ }_{n}\right), \partial\left(f \quad \varpi, f \quad \beta^{*}{ }_{n}\right)\right\}=\Gamma, \Gamma \geq 0$.

Therefore as $n \rightarrow+\infty$ in Eq. (45), we get

$$
\hat{\phi}(\Gamma) \leq \hat{\phi}(\Gamma)-\hat{\psi}(\Gamma),
$$

which we have derived $\hat{\psi}(\Gamma)=0$. Hence, $\Gamma=0$. Therefore,

$$
\lim _{n \rightarrow+\infty} \max \left\{\partial\left(f \quad \zeta, f \quad \alpha^{*}\right), \partial\left(f \quad \varpi, f \quad \beta^{*}{ }_{n}\right)\right\}=0 .
$$

Thus,

$$
\lim _{n \rightarrow+\infty} \partial\left(f \zeta \text {, } f \alpha^{*}{ }_{n}\right)=0 \text { and } \lim _{n \rightarrow+\infty} \partial\left(f \quad \varpi, f \beta^{*}{ }_{n}\right)=0 .
$$

Also from the above same argument, we procured that

$$
\lim _{n \rightarrow+\infty} \partial\left(f \quad k \text {, f } \alpha^{*}{ }_{n}\right)=0 \text { and } \lim _{n \rightarrow+\infty} \partial\left(f \quad \ell \text {,f } \beta^{*}{ }_{n}\right)=0 \text {. }
$$

Therefore from (47) and (48), we get $f \zeta=f k$ and $f \varpi=f \ell$. Since $f \zeta=\mathscr{L}(\zeta, \varpi)$ and f $\varpi=\mathscr{L}(\varpi, \zeta)$ and the commutativity property of $\mathscr{L}$ and $f$ implies that

$$
\begin{aligned}
f(f \zeta) & =f(\mathscr{L}(\zeta, \varpi))=\mathscr{L}(f \zeta, f \varpi) \text { and } f(f \varpi) \\
& =f(\mathscr{L}(\varpi, \zeta))=\mathscr{L}(f \varpi, f \zeta) .
\end{aligned}
$$

If $f \zeta=\alpha^{*}$ and $f \quad \varpi=\beta^{*}$ then from (49), we get

$$
f\left(\alpha^{*}\right)=\mathscr{L}\left(\alpha^{*}, \beta^{*}\right) \text { and } f\left(\beta^{*}\right)=\mathscr{L}\left(\beta^{*}, \alpha^{*}\right),
$$

this shows that $\left(\alpha^{*}, \beta^{*}\right)$ is a coupled coincidence point of $\mathscr{L}$ and $f$. Hence, of $\left(\alpha^{*}\right)=f k$ and $f\left(\beta^{*}\right)=f \ell$ which in turn gives that $f\left(\alpha^{*}\right)=\alpha^{*}$ and $f\left(\beta^{*}\right)=\beta^{*}$. Therefore, we conclude from (50) that $\left(\alpha^{*}, \beta^{*}\right)$ is a coupled common fixed point of $\mathscr{L}$ and $f$.

Assume $\left(\alpha^{*}, \beta^{*}\right)$ is another coupled common fixed point to $\mathscr{L}$ and $f$. Thus $\alpha^{*}=f \alpha^{*}=\mathscr{L}\left(\alpha^{*}, \beta^{*}\right)$ and $\beta^{*}=f \beta^{*}=\mathscr{L}\left(\beta^{*}, \alpha^{*}\right)$. But $\left(\alpha^{*}, \beta^{*}\right)$ is a coupled common fixed point of $\mathscr{L}$ and $f$ then $f \alpha^{*}=f \zeta=\alpha^{*}$ and if $\beta^{*}=$ ff $\varpi=\beta^{*}$. Therefore, $\alpha^{*}=$ of $\alpha^{*}=f \alpha^{*}=\alpha^{*}$ and $\beta^{*}=f \beta^{*}=f \beta^{*}=\beta^{*}$. Hence the uniqueness.

Theorem 2.12 If $f \zeta_{0} \preceq f \varpi_{0}$ or $f \zeta_{0} \succeq f \varpi_{0}$ in Theorem 2.11, then $\mathscr{L}$ and $f$ have a unique common fixed point in $\mathfrak{P}$. 
Proof Assume that $(\zeta, \varpi) \in \mathfrak{P}$ is a unique coupled common fixed point of $\mathscr{L}$ and $f$. Next to show that $\zeta=\varpi$. Suppose that $f \zeta_{0} \preceq f \varpi_{0}$ then by induction, we get $f \zeta_{n} \preceq f \varpi_{n}$ for all $n \geq 0$. From Lemma 2 of [16], we have

$$
\begin{aligned}
& \hat{\phi}\left(\sigma^{k-2} \partial(\zeta, \varpi)\right) \\
& =\hat{\phi}\left(\sigma^{k} \frac{1}{\jmath^{2}} \partial(\zeta, \varpi)\right) \\
& \leq \lim _{n \rightarrow+\infty} \sup \hat{\phi}\left(\jmath^{k} \partial\left(\zeta_{n+1}, \varpi_{n+1}\right)\right) \\
& =\lim _{n \rightarrow+\infty} \sup \hat{\phi}\left({ }^{k} \partial\left(\mathscr{L}\left(\zeta_{n}, \varpi_{n}\right), \mathscr{L}\left(\varpi_{n}, \zeta_{n}\right)\right)\right) \\
& \leq \lim _{n \rightarrow+\infty} \sup \hat{\phi}\left(\mathcal{C}_{f}\left(\zeta_{n}, \varpi_{n}, \varpi_{n}, \zeta_{n}\right)\right) \\
& -\lim _{n \rightarrow+\infty} \inf \hat{\psi}\left(\mathcal{D}_{f}\left(\zeta_{n}, \varpi_{n}, \varpi_{n}, \zeta_{n}\right)\right) \\
& \leq \hat{\phi}(ð(\zeta, \varpi)) \\
& -\lim _{n \rightarrow+\infty} \inf \hat{\psi}\left(\mathcal{D}_{f}\left(\zeta_{n}, \varpi_{n}, \varpi_{n}, \zeta_{n}\right)\right) \\
& <\hat{\phi}(ð(\zeta, \varpi)),
\end{aligned}
$$

which is a contradiction. Hence, $\zeta=\varpi$.

The result can also be seen in the case of of $\zeta_{0} \succeq f \varpi_{0}$.

Note 1 The same conclusions can also be seen as in Theorems 2.6, 2.7, 2.9, 2.11 and 2.12 by maintaining only $\mathcal{C}_{f}(\zeta, \varpi), \mathcal{C}_{f}(\zeta, \varpi, \varrho, \sigma)$ in place of $\mathcal{D}_{f}(\zeta, \varpi)$, $\mathcal{D}_{f}(\zeta, \varpi, \varrho, \sigma)$ in the contraction conditions.

Remark 2.13 Although $\delta=1$ and as a consequence of [14], the condition

$$
\begin{aligned}
& \hat{\phi}(\partial(\mathscr{L}(\zeta, \varpi), \mathscr{L}(\varrho, \varpi))) \\
& \leq \hat{\phi}(\max \{\partial(f \quad \zeta, f \quad \varrho), \partial(f \quad \varpi, f \quad \varpi)\}) \\
& -\hat{\psi}(\max \{\partial(f \quad \zeta, f \quad \varrho), \partial(f \quad \varpi, f \quad \varpi)\})
\end{aligned}
$$

is equivalent to,

$$
\begin{aligned}
& \partial(\mathscr{L}(\zeta, \varpi), \mathscr{L}(\varrho, \varpi)) \\
& \leq \varphi(\max \{\partial(f \quad \zeta, f \varrho), \partial(f \varpi, f \varpi)\}),
\end{aligned}
$$

where $\hat{\phi} \in \hat{\Phi}, \hat{\psi} \in \hat{\Psi}$ and $\varphi$ is a continuous self mapping on $[0,+\infty)$ with $\varphi(\varepsilon)<\varepsilon$ for all $\varepsilon>0$ and $\varphi(\varepsilon)=0$ if and only if $\varepsilon=0$. As a result, the findings are generalized and expanded the results of $[9,12,17]$ as well as several other comparable results.

Now depending on the continuity of a metric $\partial$, we have the following examples.
Example 2.14 Let $\mathfrak{P}=\{a, b, c, d, e, f\}$ and $\precsim: \mathfrak{P} \times \mathfrak{P} \rightarrow \mathfrak{P}$ be a metric defined by

$$
\begin{aligned}
\partial(\zeta, \varpi) & =\partial(\varpi, \zeta)=0, \text { if } \zeta=\varpi \\
& =a, b, c, d, e, f \text { and } \zeta=\varpi, \\
\partial(\zeta, \varpi) & =\partial(\varpi, \zeta)=3, \text { if } \zeta=\varpi \\
& =a, b, c, d, e \text { and } \zeta \neq \varpi, \\
\partial(\zeta, \varpi) & =\partial(\varpi, \zeta)=12, \text { if } \zeta \\
& =a, b, c, d \text { and } \varpi=f, \\
\partial(\zeta, \varpi) & =\partial(\varpi, \zeta)=20, \text { if } \zeta=e \text { and } \varpi \\
& =f, \text { with usual order } \leq .
\end{aligned}
$$

A self mapping $\mathscr{L}$ on $\mathfrak{P}$ defined by $\mathscr{L} a=\mathscr{L} b=\mathscr{L} c=\mathscr{L} d=\mathscr{L} e=1, \mathscr{L} f=2$ has a fixed point with $\hat{\phi}(\varepsilon)=\frac{\varepsilon}{2}$ and $\hat{\psi}(\varepsilon)=\frac{\varepsilon}{4}$ where $\varepsilon \in[0,+\infty)$.

Proof For $s=2,(\mathfrak{P}, \widetilde{\partial}, \leq)$ is a complete partially ordered $b$-metric space. Assume that $\zeta<\varpi$ for $\zeta, \varpi \in \mathfrak{P}$, then we have the following cases:

Case 1 If $_{\zeta, \varpi \in\{a, b, c, d, e\}}$ then $\partial(\mathscr{L} \zeta, \mathscr{L} \varpi)=\partial(a, a)=0$. Thus,

$$
\hat{\phi}(2 ð(\mathscr{L} \zeta, \mathscr{L} \varpi))=0 \leq \hat{\phi}(\mathcal{C}(\zeta, \varpi))-\hat{\psi}(\mathcal{D}(\zeta, \varpi)) .
$$

Case 2 If $\zeta \in\{a, b, c, d, e\}$ and $\varpi=f$, then $\partial(\mathscr{L} \zeta, \mathscr{L} \varpi)=\partial(a, b)=3, \mathcal{C}(f, e)=\mathcal{D}(f, e)=20$ and $\mathcal{C}(\zeta, f)=\mathcal{D}(\zeta, f)=12$, for $\zeta \in\{a, b, c, d\}$. Hence,

$$
\hat{\phi}(2 ð(\mathscr{L} \zeta, \mathscr{L} \varpi)) \leq \frac{\mathcal{C}(\zeta, \varpi)}{d}=\hat{\phi}(\mathcal{C}(\zeta, \varpi))-\hat{\psi}(\mathcal{D}(\zeta, \varpi)) .
$$

As a result, all the conditions of Theorem 2.1 are satisfied, and hence $\mathscr{L}$ has a fixed point in $\mathfrak{P}$.

Example 2.15 Define a metric $\partial$ with usual order $\leq$ by

$$
\partial(\zeta, \varpi)= \begin{cases}0, & \text { if } \zeta=\varpi \\ 1, & \text { if } \zeta \neq \varpi \in\{0,1\} \\ |\zeta-\varpi|, & \text { if } \zeta, \varpi \in\left\{0, \frac{1}{2 n}, \frac{1}{2 m}: n \neq m \geq 1\right\} \\ 6, & \text { otherwise. }\end{cases}
$$

where $\mathfrak{P}=\left\{0,1, \frac{1}{2}, \frac{1}{3}, \frac{1}{4}, \cdots \frac{1}{n}, \cdots\right\}$. Then a self mapping $\mathscr{L}$ on $\mathfrak{P}$ by $\mathscr{L} 0=0, \mathscr{L} \frac{1}{n}=\frac{1}{12 n}(n \geq 1)$ has a fixed point with $\hat{\phi}(\varepsilon)=\varepsilon$ and $\hat{\psi}(\varepsilon)=\frac{4 \varepsilon}{5}$ for $\varepsilon \in[0,+\infty)$.

Proof $\partial$ is evidently discontinuous, and $(\mathfrak{P}, \check{\partial}, \leq)$ is a complete partially ordered $b$-metric space with $\delta=\frac{12}{5}$. Now we have the following cases for $\zeta, \varpi \in \mathfrak{P}$ with $\zeta<\varpi:$ 
Case 1 Suppose $\zeta=0$ and $\sigma=\frac{1}{n}(n>0)$, then $ð(\mathscr{L} \zeta, \mathscr{L} \varpi)=\succsim\left(0, \frac{1}{12 n}\right)=\frac{1}{12 n}$ and $\mathcal{C}(\zeta, \varpi)$ or $\mathcal{D}(\zeta, \varpi)=\frac{1}{n}$ and $\mathcal{C}(\zeta, \varpi)$ or $\mathcal{D}(\zeta, \varpi)=\{1,6\}$. Therefore,

$$
\begin{aligned}
& \hat{\phi}\left(\frac{12}{5} ð(\mathscr{L} \zeta, \mathscr{L} \varpi)\right) \leq \frac{\mathcal{C}(\zeta, \varpi)}{5} \\
& =\hat{\phi}(\mathcal{C}(\zeta, \varpi))-\hat{\psi}(\mathcal{D}(\zeta, \varpi)) .
\end{aligned}
$$

Case 2 Suppose that $\zeta=\frac{1}{m}$ and $\varpi=\frac{1}{n}$ where $m>n \geq 1$, then

$$
\begin{aligned}
& \partial(\mathscr{L} \zeta, \mathscr{L} \varpi) \\
& =\partial\left(\frac{1}{12 m}, \frac{1}{12 n}\right), \mathcal{C}(\zeta, \varpi) \\
& =\mathcal{D}(\zeta, \varpi) \geq \frac{1}{n}-\frac{1}{m} \text { or } \mathcal{C}(\zeta, \varpi) \\
& =\mathcal{D}(\zeta, \varpi)=6 .
\end{aligned}
$$

Thus,

$$
\begin{aligned}
& \hat{\phi}\left(\frac{12}{5} ð(\mathscr{L} \zeta, \mathscr{L} \varpi)\right) \leq \frac{\mathcal{C}(\zeta, \varpi)}{5} \\
& =\hat{\phi}(\mathcal{C}(\zeta, \varpi))-\hat{\psi}(\mathcal{D}(\zeta, \varpi)) .
\end{aligned}
$$

Hence, we have the conclusion from Theorem 2.1 as all assumptions are fulfilled.

Example 2.16 Let $d: \mathfrak{P} \times \mathfrak{P} \rightarrow \mathfrak{P}$ be a metric with $\mathfrak{P}=\left\{\mho / \mho:\left[a_{1}, a_{2}\right] \rightarrow\left[a_{1}, a_{2}\right]\right.$ continuous $\}$ and

$$
\partial\left(\mho_{1}, \mho_{2}\right)=\sup _{\varepsilon \in\left[a_{1}, a_{2}\right]}\left\{\left|\mho_{1}(\varepsilon)-\mho_{2}(\varepsilon)\right|^{2}\right\}
$$

for every $\mho_{1}, \mho_{2} \in \mathfrak{P}, 0 \leq a_{1}<a_{2}$ with $\mho_{1} \preceq \mho_{2}$ implies $a_{1} \leq \mho_{1}(\varepsilon) \leq \mho_{2}(\varepsilon) \leq a_{2}, \varepsilon \in\left[a_{1}, a_{2}\right]$. A self mapping $\mathscr{L}$ on $\mathfrak{P}$ defined by $\mathscr{L} \mho=\frac{\mho}{5}, \mho \in \mathfrak{P}$ has a unique fixed point with $\hat{\phi}(\ddot{a})=\ddot{a}$ and $\hat{\psi}(\ddot{a})=\frac{\ddot{a}}{3}$, for any $\ddot{a} \in[0,+\infty]$.

Proof Since, $\min \left(\mho_{1}, \mho_{2}\right)(\varepsilon)=\min \left\{\mho_{1}(\varepsilon), \mho_{2}(\varepsilon)\right\} \quad$ is continuous, and all other assumptions of Theorem 2.3 are satisfied for $s=2$. As a result, $0 \in \mathfrak{P}$ is the only fixed point of $\mathscr{L}$.

\section{Limitations}

In complete partially ordered $b$-metric space, the existence and uniqueness of a fixed point for a self mapping which satisfies a generalized weak contraction condition with two rational auxiliary functions are discussed. These results are further generalized for two self mappings in the same context and proved the existence of coincidence point, coupled coincidence point and coupled common fixed points. Also, shown that these results are generalized the well known existing results in the literature. Some numerical examples are given to justify the obtained results.

- These results can be extended by involving more mappings in partially ordered $b$-metric space to acquire triple, quadruple fixed points.

- These contractions can be used to obtain a coincidence point, coupled coincidence point and coupled common fixed points for the mappings in various ordered metric spaces with required topological properties like monotone non-decreasing, mixed monotone, compatible etc.

\section{Acknowledgements}

The authors do thankful to the editor for giving and opportunity to submit our research paper in this esteemed journal.

\section{Authors' contributions}

KK involved in formal analysis, methodology, writing and supervising the work. NSR contributed in the conceptualization, formal analysis, methodology, writing, editing and approving the manuscript. Both authors read and approved the final manuscript.

\section{Funding}

Not applicable.

Availability of data and materials

Not applicable.

\section{Declarations}

Ethics approval and consent to participate

Not applicable.

\section{Consent for publication}

Not applicable.

\section{Competing interests}

The authors declare that they have no competing interests.

\section{Author details}

${ }^{1}$ Department of Mathematics, Vignan's Foundation for Science, Technology \& Research, Vadlamudi, Andhra Pradesh 522213, India. ${ }^{2}$ Department of Applied Mathematics, School of Applied Natural Sciences, Adama Science and Technology University, Post Box No.1888, Adama, Ethiopia.

Received: 21 November 2021 Accepted: 24 January 2022

Published online: 09 February 2022

\section{References}

1. Aghajani A, Arab R. Fixed points of $(\psi, \phi, \theta)$-contractive mappings in partially ordered $b$-metric spaces and applications to quadratic integral equations. Fixed Point Theory Appl. 2013;2013:245.

2. Aghajani A, Abbas M, Roshan JR. Common fixed point of generalized weak contractive mappings in partially ordered $b$-metric spaces. Math Slovaca. 2014;64(4):941-60.

3. Akkouchi M. Common fixed point theorems for two self mappings of a $b$-metric space under an implicit relation. Hacet J Math Stat. 2011;40(6):805-10. 
4. Aleksić S, Huang H, Mitrović ZD, Radenović S. Remarks on some fixed point results in b-metric spaces. J Fixed Point Theory Appl. 2018;20:147. https://doi.org/10.1007/s11784-018-2.

5. Allahyari R, Arab R, Haghighi AS. A generalization on weak contractions in partially ordered $b$-metric spaces and its applications to quadratic integral equations. J Inequal Appl. 2014;2014:355.

6. Aydi H, Dedović N, Bin-Mohsin B, Filipović M, Radenović S. Some new observations on Geraghty and Ćirić type results in b-metric spaces. Mathematics. 2019;7:643. https://doi.org/10.3390/math707064.

7. Bakhtin IA. The contraction principle in quasimetric spaces. Funct Anal Ulianowsk Gos Fed Inst. 1989;30:26-37.

8. Belay M, Karusala K, Seshagiri Rao N. Some fixed point results of generalized $(\phi, \psi)$-contractive mappings in ordered $b$-metric spaces. BMC Res Notes. 2020;13:537. https://doi.org/10.1186/s13104-020-05354-1.

9. Bhaskar TG, Lakshmikantham V. Fixed point theorems in partially ordered metric spaces and applications. Nonlinear Anal. 2006;6:1379-93. https:// doi.org/10.1016/j.na.2005.10.017.

10. Czerwik S. Contraction mappings in b-metric spaces. Acta Math Univ Ostrav. 1993;1:5-11.

11. Dorić D. Common fixed point for generalized $(\psi, \phi)$-weak contractions. Appl Math Lett. 2009;22:1896-900.

12. Harjani J, López B, Sadarangani K. Fixed point theorems for mixed monotone operators and applications to integral equations. Nonlinear Anal. 2011;74:1749-60.

13. Huang H, Radenović S, Vujaković J. On some recent coincidence and immediate consequences in partially ordered $b$-metric spaces. Fixed Point Theory Appl. 2015;2015:63. https://doi.org/10.1186/s13663-015-0308-3.

14. Jachymski J. Equivalent conditions for generalized contractions on (ordered) metric spaces. Nonlinear Anal. 2011;74:768-74.

15. Jovanović M, Kadelburg Z, Radenović S. Common fixed point results in metric-type spaces. Fixed Point Theory Appl. 2010;2010:978121.

16. Karapinar E, Mitrović ZD, Özturk A, Radenović S. On a theorem of Ćirić in b-metric spaces. Rendiconti del Circolo Matematico di Palermo Series 2. https://doi.org/10.1007/s12215-020-00491-9.

17. Luong NV, Thuan NX. Coupled fixed point theorems in partially ordered metric spaces. Bull Math Anal Appl. 2010;4:16-24.

18. Roshan JR, Parvaneh V, Sedghi S, Shobkolaei N, Shatanawi W. Common fixed points of almost generalized $(\psi, \phi)_{5}$-contractive mappings in ordered b-metric spaces. Fixed Point Theory Appl. 2013;2013(159):1-23.

19. Roshan JR, Parvaneh V, Altun I. Some coincidence point results in ordered b-metric spaces and applications in a system of integral equations. Appl Math Comput. 2014;226:725-37.

20. Seshagiri Rao N, Kalyani K. Fixed point theorems for nonlinear contractive mappings in ordered $b$-metric space with auxiliary function. BMC Res Notes. 2020;13:451. https://doi.org/10.1186/s13104-020-05273-1.

21. Seshagiri Rao N, Kalyani K. Generalized contractions to coupled fixed point theorems in partially ordered metric spaces. J Sib Federal Univ Math Phys. 2020;13(4):492-502. https://doi.org/10.17516/ 1997-1397-2020-13-4-492-502.

22. Seshagiri Rao N, Kalyani K. Coupled fixed point theorems with rational expressions in partially ordered metric spaces. J Anal. 2020. https://doi. org/10.1007/s41478-020-00236-y.

23. Seshagiri Rao N, Kalyani K, Khatri K. Contractive mapping theorems in partially ordered metric spaces. CUBO. 2020;22(2):203-14.

24. Seshagiri Rao N, Kalyani K. Generalized fixed point results of rational type contractions in partially ordered metric spaces. BMC Res Notes 2021;14:390. https://doi.org/10.1186/s13104-021-05801-7.

25. Seshagiri Rao N, Kalyani K, Prasad K. Fixed point results for weak contractions in partially ordered $b$-metric space. BMC Res Notes. 2021;14:263. https://doi.org/10.1186/s13104-021-05649-x.

\section{Publisher's Note}

Springer Nature remains neutral with regard to jurisdictional claims in published maps and institutional affiliations.

Ready to submit your research? Choose BMC and benefit from:

- fast, convenient online submission

- thorough peer review by experienced researchers in your field

- rapid publication on acceptance

- support for research data, including large and complex data types

- gold Open Access which fosters wider collaboration and increased citations

- maximum visibility for your research: over $100 \mathrm{M}$ website views per year

At BMC, research is always in progress.

Learn more biomedcentral.com/submissions 